\title{
Low Test Reference Range
}

National Cancer Institute

\section{Source}

National Cancer Institute. Low Test Reference Range. NCI Thesaurus. Code C70934.

Reported low limit of the test reference range. 